\title{
ABBREVIATIONS AND SYMBOLS
}

\section{BIBLIOGRAPHICAL ABBREVIATIONS}

$B D B \quad$ F. Brown, S. R. Driver and C. A. Briggs, A Hebrew and English Lexicon of the Old Testament (Oxford: Clarendon Press, 1976).

BSOAS Bulletin of the School of Oriental and African Studies. Compendious J. Payne Smith, A Compendious Syriac Dictionar (Oxford: Clarendon Press 1979).

DNWSI J. Hoftijzer and K. Jongeling, Dictionary of the NorthWest Semitic Inscriptions, 2 vols. (Handbuch der Orientalistik I/XXI.1-2; Leiden: E. J. Brill, 1995).

Hatra Published Hatran Inscriptions.

Ibn Doreid F. Wüstenfeld, Abu Bekr Muhammed ben el-Hassan Ibn Doreid's genealogisch-etymologisches Handbuch (Göttingen: Dieterichsche Buchhandlung, 1854).

Lane $\quad$ E. W. Lane, An Arabic-English Lexicon, 8 parts (London: Williams and Norgate, 1863-1893).

OSI H. J. W. Drijvers and J. F. Healey, The Old Syriac Inscriptions of Edessa and Osrhoene (Handbuch der Orientalistik, I/XLII; Leiden: E.J. Brill, 1999).

Thes. Syr. $\quad$ R. Payne Smith, Thesaurus Syriacus, 2 vols. (Oxford: Clarendon Press, 1879-1901).

ZDMG Zeitschrift der Deutschen Morgenländischen Gesellschaft

\section{GenERAL ABBREVIATIONS}

abs. absolute state

act. part. active participle 
adj. adjective

Akk. Akkadian

Anc. Aram. Ancient Aramaic

Arab. Arabic

B. Aram. Biblical Aramaic

class. Syr. classical Syriac

constr. construct state

D. $\quad$ refers to the numbers of the Syriac inscriptions in H. J. W. Drijvers, Old Syriac (Edessean) Inscriptions (Semitic Studies Series 3; Leiden, E. J. Brill, 1972).

DP. Duro-Europos Parchment in Drijvers 1972

Emp. Aram. Emperial Aramaic

emph. emphatic state

fem. feminine

Hat. Hatran

Heb. Hebrew

imperf. imperfect

inf. Infinitive

J. Aram. Jewish Aramaic

masc. masculine

n. (in footnotes) note

Nab. Nabataean

P. Parchment

P. Dura Parchment found at Dura-Europos

P. Euphr. Parchments and Papyri found in the Middle Euphrates area

Palm. Palmayrene

pass. part. passive participle

perf. Perfect

Ph. Phoenician

Pun. Punic

sing. Singular

suff. Suffix

Ug. Ugaritic 


\section{SYMBOLS}

$\begin{array}{ll}{[\quad]} & \text { Restoration of parts now illegible or lost } \\ \ldots \ldots . . & \text { Lost or unreadable letters } \\ * & \text { Assumed form } \\ ? & \text { Uncertain letters } \\ > & \text { Developed to } \\ < & \text { Developed from }\end{array}$


